\title{
A GENERAL THEORY OF ONE-DIMENSIONAL LOCAL RINGS
}

\author{
by D. G. NORTHCOTT \\ (Received 24th February, 1955)
}

\begin{abstract}
Introduction. The development of the theory of local rings has been greatly stimulated by the importance of the applications to algebraic geometry, but it is none the less true that this stimulus has produced a theory which, on aesthetic grounds, is somewhat unsatisfactory. In the first place, if a local ring $Q$ arises in the ordinary way from a geometric problem, then $Q$ will have the same characteristic as its residue field. It is partly for this rcason that our knowledge of equicharacteristic local rings is much more extensive than it is of those local rings which present the case of unequal characteristics. Again, in the geometric case, the integral closure of $Q$ in its quotient field will be a finite $Q$-module. Here, once more, we have a special situation which it would be desirable to abandon from the point of view of a general abstract theory.
\end{abstract}

In recent years, substantial progress has been made towards the construction of a theory of local rings freed from the restrictions suggested by geometry. There are still many unsolved problems in this field, but if one considers only one-dimensional local rings then a substantial body of results is known, which form, in a sense, a complete unit. The present paper is an expository account of these results. It contains, in particular, an investigation of the relations which hold between a one-dimensional local ring and the discrete rank 1 valuations associated with it. This corresponds, of course, to the theory which connects a point $P$ of a curve with the places of the curve having $P$ as centre. In this connection, it may be noted that the main result of the paper (Theorem 6 ) is essentially a generalisation of the theorem $\dagger$ which asserts that an intersection multiplicity of two curves (as defined, in a wellknown way, by means of " places ") involves the two curves symmetrically.

It is inevitable, because the basic concepts involved are both simple and familiar, that the literature should contain many discussions which are connected with the one given here, and indeed examples can be found which go back more than twenty years. However, among all the original contributions to this subject, the paper of Krull (5) entitled Ein Satz über primäre Integritätsbereiche is of particular significance. The central result of Krull's paper is effectively our Theorem 7, and this contains the unexpected element of the investigation. Given Krull's theorem, many of the other results are to a certain extent inevitable, but the proofs contain a number of interesting features which will well repay a closer examination.

1. Grell's theorem on lengths of primary ideals. The letter $Q$ will be used to denote a local ring, and $m$ will denote its maximal ideal. The proposition which follows contains an elementary observation concerning local rings of dimension unity. The formal proof is omitted.

Proposition 1. For a local ring of dimension unity, every element of $m$ is a zero-divisor if and only if $\mathrm{m}$ belongs to the zero ideal. If there are elements of 11 which are not zero-divisors, then all the prime ideals belonging to the zero ideal are of first dimension; in this case, a proper ideal is $\mathrm{m}$-primary if and only if it contains an element which is not a zero-divisor.

In any particular theorem, our hypotheses will usually ensure that we are not dealing

† See Walker (10), Chapter IV, Theorem 5.1. 
with a local ring in which the maximal ideal is composed exclusively of zero-divisors, even if this assumption is not made explicitly.

Proposition 2. Suppose that $\operatorname{dim} Q=1$, that $x$ belongs to $\mathrm{m}$ and is not a zcro-divisor, and that $\mathrm{a}$ is an m-primary ideal. Then ax is m-primary and

$$
\text { length }(\mathfrak{a} x)=\text { length } \mathfrak{a}+\text { length }(x) \text {. }
$$

Further, length $(x)$ is equal to the length of a composition series of ideals from a to ax.

Proof. If we regard $Q$ and $Q x$ as $Q$-modules then the mapping $q \rightarrow q x$ defines a $Q$-homomorphism of $Q$ on to $Q x$. Since $x$ is not a zero-divisor, the inverse image of ax is simply a and therefore there is a $1-1$ correspondence relating the ideals between $Q$ and $a$ to those between $Q x$ and $a x$. Accordingly,

$$
\text { length } a=\text { length } a x \text { - length }(x) \text {, }
$$

which proves the first assertion. Since the relation just proved can be rewritten as

$$
\text { length }(x)=\text { length } a x \text {-length } \mathfrak{a} \text {, }
$$

the second assertion follows.

Corollary. If $\operatorname{dim} Q=1, x$ belongs to $\mathrm{m}$ and is not a zero divisor, then

$$
\text { length }\left(x^{n}\right)=n \text { length }(x) \text {. }
$$

For length $\left(x^{n}\right)=$ length $\left(Q x^{n-1} \cdot x\right)=$ length $(x)+$ length $\left(x^{n-1}\right)$

by the Proposition, and on repeating the argument the Corollary follows. Incidently we see that the length of $(x)$ is equal to its multiplicity in the sense of Samuel (see (9), Chapter II, 2).

To avoid overburdening the statement of the main result of this section, we shall make some preliminary remarks. Let $Q$ be a one-dimensional local ring and denote by $S$ its full ring of quotients. Further, let $P$ be any extension ring of $Q$ which is contained in $S$ and which is a finite $Q$-module. In these circumstances there is a conductor $f$ from $Q$ to $P$ consisting of all elements $\alpha$ belonging to $S$ such that $\alpha P \subseteq Q$. Concerning $f$ it should be noted that, besides being contained in $Q$, it is both a $Q$-ideal and a $P$-ideal. Moreover, wc can find an element of $f$ which is not a zero-divisor, consequently either $\mathfrak{f}$ is m-primary or $f=Q$.

The ring $P$ will be a semi-local ring; let $p_{1}, \mathfrak{p}_{2}, \ldots, \mathfrak{p}_{l}$ be its maximal ideals. If now $x$ belongs to $m$ and is not a zero-divisor, then the prime ideals which belong to $P x$ are just $\mathfrak{p}_{1}, \mathfrak{p}_{2}, \ldots, \mathfrak{p}_{l}$. This shows that a normal decomposition of $P x$ can, because the $p_{i}$ are maximal, be written in either of the forms

$$
P x=\mathfrak{q}_{1} \cap \mathfrak{q}_{2} \cap \ldots \cap \mathfrak{q}_{l}=\mathfrak{q}_{1} \mathfrak{q}_{2} \ldots \mathfrak{q}_{l}
$$

where $\mathfrak{q}_{i}$ is $\mathfrak{p}_{i}$-primary. Here the components $\mathfrak{q}_{i}$ are unique because the $\mathfrak{p}_{i}$ will be minimal prime ideals of $P x$. We make use of these observations and also of these notations in

THEOREM 1 (Grell $\dagger$ ). Suppose that $x$ belongs to $\mathrm{m}$ and is not a zero-divisor in $Q$, and let

$$
P x=\mathfrak{q}_{1} \mathfrak{q}_{2} \ldots \mathfrak{q}_{l}
$$

where $\mathfrak{q}_{i}$ is $\mathfrak{p}_{i}$-primary. Then

$$
\text { length } \left.(Q x)=\sum_{i=1}^{l} \text { (length } \mathfrak{q}_{i}\right)\left[P / \mathfrak{p}_{i}: Q / \mathrm{m}\right]
$$

Proof. Choose $n$ so large that $x^{n} \in \mathfrak{f}$; then

$$
P x^{n+1}=q_{1}^{n+1} \mathfrak{q}_{2}^{n+1} \ldots \mathfrak{q}_{l}^{n+1} \subseteq P x^{n}=q_{1}^{n} \mathfrak{q}_{2}^{n} \ldots \mathfrak{q}_{l}^{n} \subseteq \mathfrak{f} \subseteq Q .
$$

$\dagger$ A theorem of very similar character but with rather different assumptions, is due originally to Grell (4). 
We shall first consider a composition series of $P$-ideals from

Let

$$
\mathfrak{q}_{1}^{n+1} q_{2}^{n+1} \ldots q_{l}^{n+1} \text { to } q_{1}^{n} q_{2}^{n} \ldots q_{l}^{n} .
$$

be a composition series of $\mathfrak{p}_{1}$-primary ideals ; then

$$
q_{1}^{n}=q_{10} \supset q_{11} \supset q_{12} \supset \ldots \supset q_{1 t}=q_{1}^{n+1}
$$

$$
\mathfrak{q}_{10} \mathfrak{q}_{2}^{n+1} \mathfrak{q}_{3}^{n+1} \ldots \mathfrak{q}_{l}^{n+1}, \mathfrak{q}_{11} \mathfrak{q}_{2}^{n+1} \mathfrak{q}_{3}^{n+1} \ldots \mathfrak{q}_{l}^{n+1}, \mathfrak{q}_{12} \mathfrak{q}_{2}^{n+1} \mathfrak{q}_{3}^{n+1} \ldots \mathfrak{q}_{l}^{n+1}, \ldots, \mathfrak{q}_{1 t} \mathfrak{q}_{2}^{n+1} \mathfrak{q}_{3}^{n+1} \ldots \mathfrak{q}_{l}^{n+1},
$$

is clearly a composition series of $P$-ideals. But all these ideals are contained in $Q$; consequently they can be regarded as $Q$-ideals as well as $P$-ideals. Of course, if we regard them as $Q$-ideals then they need no longer form a composition series. Now $\mathfrak{q}_{1 i} \mathfrak{p}_{1} \subseteq \mathfrak{q}_{1, i+1}$; hence

and therefore

$$
\left(q_{1 i} q_{2}^{n+1} \ldots q_{l}^{n+1}\right) p_{1} \subseteq q_{1, i+1} q_{2}^{n+1} \ldots q_{l}^{n+1}
$$

$$
\mathfrak{q}_{1 i} \mathfrak{q}_{2}^{n+1} \ldots \mathfrak{q}_{l}^{n+1} / \mathfrak{q}_{1, i+1} \mathfrak{q}_{2}^{n+1} \ldots \mathfrak{q}_{l}^{n+1}
$$

is a vector space over the field $R / p_{1}$. Further, since there are no $P$-ideals between $q_{1 i} q_{2}^{n+1} \ldots q_{l}^{n+1}$ and $q_{1, i+1} q_{2}^{n+1} \ldots q_{l}^{n+1}$, the dimension of this vector space is unity. It follows that as vector space over the subfield $Q / \mathrm{m}$ its dimension is $\left[P / p_{1}: Q / \mathrm{m}\right]=f_{1}$ (say) ; consequently a composition series of $Q$-ideals from $q_{1 i} q_{2}^{n+1} \ldots q_{l}^{n+1}$ to $q_{1, i+1} q_{2}^{n+1} \ldots q_{l}^{n+1}$ has length $f_{1}$. Accordingly, if we combine these results for the various possible values of $i$ we find that the length of a composition series of $Q$-ideals from $\mathfrak{q}_{1}^{n+1} \mathfrak{q}_{2}^{n+1} \ldots \mathfrak{q}_{l}^{n+1}$ to $q_{1}^{n} q_{2}^{n+1} \ldots q_{l}^{n+1}$ is

$$
\left[P / p_{1}: Q / m\right] t=\left[P / p_{1}: Q / m\right]\left[\text { length } \mathfrak{q}_{1}^{n+1} \text { - length } q_{1}^{n}\right]
$$

By means of the same device, a composition series of $Q$-ideals from

$$
\mathfrak{q}_{1}^{n} q_{2}^{n+1} q_{3}^{n+1} \ldots q_{l}^{n+1} \text { to } \mathfrak{q}_{1}^{n} q_{2}^{n} q_{3}^{n+1} \ldots \mathfrak{q}_{l}^{n+1}
$$

is seen to contain

$$
\left[P / \mathfrak{p}_{2}: Q / \mathfrak{m}\right]\left[\text { length } \mathfrak{q}_{2}^{n+1}-\text { length } \mathfrak{q}_{2}^{\mathfrak{n}}\right]
$$

terms. Continuing this process, it emerges that

$$
\sum_{i=1}^{n}\left[P / p_{i}: Q / \mathrm{m}\right]\left[\text { length } q_{i}^{n+1}-\text { length } q_{i}^{n}\right]
$$

is the length of a composition series of $Q$-ideals from

$$
P x^{n+1}=\mathfrak{q}_{1}^{n+1} \mathfrak{q}_{2}^{n+1} \ldots \mathfrak{q}_{l}^{n+1} \text { to } P x^{n}=\mathfrak{q}_{1}^{n} \mathfrak{q}_{2}^{n} \ldots \mathfrak{q}_{l}^{n} .
$$

But if $P x^{n}=a$ (say), then $P x^{n+1}=a x$ and therefore, by Prop. 2, length $(Q x)$ is the length of a composition series of $Q$-ideals from $P x^{n+1}$ to $P x^{n}$. The theorem will therefore follow if we show that

$$
\text { length } q_{i}^{n+1} \text { - length } q_{i}^{n}=\text { length } \mathfrak{q}_{i} \text {. }
$$

To establish this, let $Q_{i}^{\prime}$ be the generalised ring of quotients of $P$ with respect to $\mathfrak{p}_{i}$ (see, for example, (8) Chapter 2, $\S 2.7)$; then $Q_{i}^{\prime}$ is a one-dimensional local ring. Further, if $\mathfrak{q}_{2}=Q_{i}^{\prime} \mathfrak{q}_{i}$, then, for any integer $r$, length $\mathfrak{q}_{i}^{r}=$ length $\mathfrak{q}_{i}^{\prime r}$ and so it will suffice to show that

But from

$$
\text { length } \mathfrak{q}_{i}^{\prime n+1} \text { - length } \mathfrak{q}_{i}^{\prime n}=\text { length } \mathfrak{q}_{i}^{\prime} \text {. }
$$

$$
P x=\mathfrak{q}_{1} \cap \mathfrak{a}_{2} \cap \ldots \cap \mathfrak{q}_{l}
$$


follows

$$
Q_{i}^{\prime} x^{\prime}=\mathfrak{q}_{i}^{\prime},
$$

where $x^{\prime}$ is the residue of $x$ modulo the component of the zero ideal (of $P$ ) determined by $P-\mathfrak{p}_{i}$. Since, however, $x$ is not a zero-divisor, it follows that $x^{\prime}$ is not a zero-divisor, consequently (see Prop. 2, Cor.)

$$
\text { length }\left(x^{\prime n+1}\right) \text { - length }\left(x^{\prime n}\right)=\text { length }\left(x^{\prime}\right)
$$

and this is equivalent to (1.1). This completes the proof.

2. Effects of the structure of the zero ideal. As before, we denote by $Q$ a one-dimensional local ring having the property that not all the elements of 12 are zero-divisors. By Proposition 1, the zero ideal of $Q$ is an unmixed ideal and

$$
Q 0=\mathfrak{u}_{1} \cap n_{2} \cap \ldots \cap n_{l}
$$

where $n_{i}$ is a primary ideal belonging to a one-dimensional prime ideal. If now $S$ is the full ring of quotients of $Q$ and $2 \eta_{i}=S n_{i}$, then

$$
50=2 \pi_{1} \frown 2 \tau_{2} \frown \ldots \frown 2 \pi_{l}
$$

is a normal primary decomposition of the zero ideal of $\subseteq$. Further, since $2 \tau_{i}+2 \tau_{j}=5$ if $i \neq j$, we can find elements $e_{1}, e_{2}, \ldots, e_{l}$ such that $e_{i} \equiv 1\left(\bmod 2 \tau_{i}\right)$ and $e_{i} \equiv 0\left(\bmod 2 \tau_{j}\right)$ whenever $i \neq j$. These elements are uniquely determined by these requirements and they have the further properties

$$
e_{1}+e_{2}+\ldots+e_{l}=1, \quad e_{i}^{2}=e_{i}, \text { and } e_{i} e_{j}=0 \text { for } i \neq j .
$$

If therefore we put

$$
P=Q e_{1}+Q e_{2}+\ldots+Q e_{l},
$$

then $P$ will be an extension ring of $Q$ as well as a finite $Q$-module and so we shall be able to apply Theorem 1 to this ring. To prepare the way for the application, we write $\tilde{\pi}_{i}=2 \tau_{i} \cap P$ and note that this makes

$$
P 0=\tilde{n}_{1} \cap \tilde{n}_{2} \cap \ldots \cap \tilde{n}_{l}
$$

a normal primary decomposition of the zero ideal of $P$.

Consider now the homomorphism of $S$ defined by the mapping $x \rightarrow x e_{i}$. This has kernel $2 \tau_{i}$ and as $2 \tau_{i} \cap Q=n_{i}$, the image $Q e_{i}$ of $Q$ under the mapping is isomorphic to $Q / n_{i}$. But $P e_{i}=Q e_{i}$; consequently $P / \tilde{n}_{i}=Q / n_{i}$. Again, if $q_{j}$ belongs to $Q$, for $1 \leqslant j \leqslant l$, and

$$
\left(q_{1} e_{1}+q_{2} e_{2}+\ldots+q_{l} e_{l}\right) e_{i}=0 \text {, }
$$

then $q_{i} e_{i}=0$, and this shows that

$$
\tilde{n}_{i}=Q e_{1}+\ldots+Q e_{i-1}+Q e_{i+1}+\ldots+Q e_{l} .
$$

We can now prove

Proposition 3. The ring $P$ has precisely $l$ maximal prime ideals $\mathfrak{p}_{1}, \mathfrak{p}_{2}, \ldots, \mathfrak{p}_{l}$ and each of these contains one and only one of the ideals $\tilde{n}_{i}$. If the $p_{i}$ are numbered so that $\tilde{n}_{i} \subseteq p_{i}$ then $\tilde{n}_{i}$ is the component of the zero ideal of $P$ determined by $P-\mathfrak{p}_{i}$.

Proof. We can certainly find at least one maximal prime ideal $\mathfrak{p}$ which contains (say) $\tilde{n}_{1}$ and, since $P / \tilde{n}_{1}=Q / \mathrm{r}_{1}$ is a local ring, there is only one such maximal ideal. Again, $p$ cannot contain any of the ideals $\tilde{\mathfrak{n}}_{2}, \tilde{n}_{3}, \ldots, \tilde{\mathfrak{n}}_{l}$. For suppose, for instance, that $\tilde{\mathfrak{n}}_{2} \subseteq \mathfrak{p}$; then, by (2.1), $e_{1}, e_{2}, \ldots, e_{l}$ are all contained in $p$. This, however, implies that $l \in \mathfrak{p}$ and we have a 
contradiction. Since every maximal ideal contains at least one of $\tilde{n}_{1}, \tilde{n}_{2}, \ldots, \tilde{n}_{l}$ this proves the first assertion, and the second assertion follows at once from the first.

Now suppose that $x$ is an element of $m$ which is not a zero-divisor and that

$$
P x=q_{1} \cap q_{2} \cap \ldots \cap q_{l}=q_{1} q_{2} \ldots q_{l}
$$

is a resolution of $P x$ into primary components. By Theorem 1,

$$
\text { length }(Q x)=\Sigma\left(\text { length } q_{i}\right)\left[P / p_{i}: Q / \mathrm{m}\right]
$$

but, since $P / \tilde{n}_{i}=Q / \mathrm{r}_{i}$, in the present case $\left[P / p_{i}: Q / \mathrm{m}\right]=1$. Further, since $\tilde{\pi}_{i}$ is the component of the zero ideal determined by $P-p_{i}$, it is contained in any $p_{i}$-primary ideal and therefore, in particular, $\tilde{n}_{i} \subseteq q_{i}$. This shows that length $q_{i}=$ length $\left(q_{i} / \tilde{n}_{i}\right)$. Now if $i \neq j$, then $q_{j}+\tilde{n}_{i}=P$ because $\mathfrak{p}_{j}$ does not contain $\tilde{n}_{i}$. Consequently, if $x_{i}$ denotes the residue of $x$ modulo $\tilde{n}_{i}$, we see that $\mathfrak{q}_{i} / \tilde{n}_{i}$ is generated by $x_{i}$ in $P / \tilde{n}_{i}$. we have

But $P / \tilde{n}_{i}=Q / \|_{i} ;$ accordingly $\mathfrak{q}_{i} / \tilde{n}_{i}$ is the principal ideal generated by $x_{i}$ in $Q / n_{i}$ and so

and therefore

$$
\text { length } \mathfrak{q}_{i}=\text { length }\left(\mathfrak{q}_{i} / \tilde{n}_{i}\right)=\text { length }\left\{\left(Q / \mathfrak{n}_{i}\right) x_{i}\right\}
$$

$$
\text { length }(Q x)=\sum_{i=1}^{l} \text { length }\left\{\left(Q / \mathrm{n}_{i}\right) x_{i}\right\}
$$

This result is restated in

TheOREM 2. Let $x$ be an element of $\mathrm{m}$ which is not a zero-divisor and let

$$
Q 0=\mathrm{n}_{1} \cap \mathrm{n}_{2} \cap \ldots \cap \mathrm{n}_{l}
$$

be a normal primary decomposition of the zero ideal. If now $x_{i}$ denotes the residue of $x$ modulo $n_{i}$, then

$$
\text { length }(Q x)=\sum_{i=1}^{l} \text { length }\left\{\left(Q / n_{i}\right) x_{i}\right\}
$$

3. Further effects of the structure of the zero ideal. The rings $Q / n_{i}$, which appear in the statement of Theorem 2, are such that their zero ideals are all of them primary ideals and so, in the Theorem which follows, we turn our attention to one-dimensional local rings which exhibit this property.

Theorem 3. Let $Q$ be a one-dimensional local ring whose zero ideal is a primary ideal belonging (say) to the prime ideal $\mathrm{p}$. Let $x$ be an element of $\mathrm{m}$ which is not a zero-divisor and, finally, let $x^{\prime}$ denote the residue of $x$ modulo $p$. Then

$$
\text { length }(Q x)=\text { length }(Q 0) \text {. length }\left\{(Q / P) x^{\prime}\right\} \text {. }
$$

Proof. We proceed by induction on the length $s$ of the zero ideal of $Q$. If $s=1$ the theorem is obvious. We therefore suppose that $s>1$ and that the theorem has been proved in the case of one-dimensional local rings whose zero ideals are primary and of length $s-1$. Let

$$
p=n_{1} \supset n_{2} \supset \ldots \supset n_{s}=(0)
$$

be a composition series of $\mathcal{P}$-primary ideals; then, by applying the inductive hypothesis to the ring $Q^{*}=Q / n_{s-1}$ and the ideal generated by the residue $x^{*}$ of $x$, we see that

$$
(s-1) \text { length }\left\{(Q / P) x^{\prime}\right\}=\text { length }\left(Q^{*} x^{*}\right)=\text { length }\left(Q x+n_{s-1}\right) \text {; }
$$

consequently it will suffice to show that

$$
\text { length }(Q x) \text { - length }\left(Q x+n_{s-1}\right)=\text { length }\left\{(Q / P) x^{\prime}\right\} \text {. }
$$


But we have the following equalities relating the lengths of certain $Q$-modules, namely

consequently it will suffice to prove that

$$
\text { length } \begin{aligned}
(Q x)-\text { length }\left(Q x+n_{s-1}\right) & =\text { length }\left(\frac{Q x+n_{s-1}}{Q x}\right)=\text { length }\left(\frac{n_{s-1}}{Q x \cap n_{s-1}}\right) \\
& =\text { length }\left(\frac{n_{s-1}}{x n_{s-1}}\right) ;
\end{aligned}
$$

$$
\text { length }\left(\frac{n_{s-1}}{x n_{s-1}}\right)=\text { length }\left(Q^{\prime} x^{\prime}\right)
$$

where $Q^{\prime}=Q / \mathcal{P}$. Choose $\theta \in \boldsymbol{n}_{s-1}$ so that $\theta \neq 0$; then the $\mathcal{P}$-primary component of $Q \theta$ is $\boldsymbol{n}_{s-1}$ and therefore we can find $c \notin \mathcal{P}$ for which $c \mathrm{n}_{s-1} \subseteq Q \theta$. We then have

$$
c n_{s-1} \subseteq Q \theta \subseteq \mathrm{u}_{s-1}
$$

and, by consideration of the mapping $q \rightarrow q c$,

$$
\text { length }\left(\frac{c \mathfrak{n}_{s-1}}{x c n_{s-1}}\right)=\text { length }\left(\frac{\mathfrak{n}_{s-1}}{x \mathfrak{n}_{s-1}}\right) \text {. }
$$

Now $n_{s-1} p=(0)$; hence $n_{s-1}$ and all the ideals contained in it form modules with respect to $Q^{\prime}=Q / P$. Indeed the ideals contained in $n_{s-1}$ and the $Q^{\prime}$-modules contained in $n_{s-1}$ are one and the same thing. Clearly $Q \theta=Q^{\prime} \theta$. Also, if $q \in Q$, then $q \theta=0$ if and only if $q \in \mathcal{P}$; con. sequently if $q^{\prime} \in Q^{\prime}$, then $q^{\prime} \theta=0$ if and only if $q^{\prime}=0$. This shows that every element of $Q \theta$ has a unique representation in the form $q^{\prime} \theta$, and we see from this that to each $Q$-ideal $b$ which is contained in $Q \theta$, there corresponds a unique $Q^{\prime}$-ideal $b^{*}$ such that $b=b^{*} \theta$. In particular $c n_{s-1}=\mathfrak{a}^{*} \theta$ for a certain non-zero $Q^{\prime}$-ideal $\mathfrak{a}^{*}$ and then $x c n_{s-1}=\left(x^{\prime} \mathfrak{a}^{*}\right) \theta$. Note that $\mathfrak{a}^{*}$ and $x^{\prime} \mathfrak{a}^{*}$ are primary ideals belonging to the maximal ideal $\mathrm{m}^{\prime}$ of $Q^{\prime}$.

To complete the proof, let

$$
\mathfrak{a}^{*}=\mathfrak{b}_{0}^{*} \supset \mathfrak{b}_{1}^{*} \supset \mathfrak{b}_{2}^{*} \supset \ldots \supset \mathfrak{b}_{r}^{*}=x^{\prime} \mathfrak{a}^{*}
$$

be a composition series of $Q^{\prime}$-ideals ; then

$$
c n_{s-1}=\mathfrak{b}_{0}^{*} \theta \supset \mathfrak{b}_{1}^{*} \theta \supset \ldots \supset \mathfrak{b}_{\tau}^{*} \theta=x c 11_{s-1}
$$

is a composition series of $Q^{\prime}$-modules and therefore of $Q$-ideals. Consequently

$$
\text { length }\left(\frac{c n_{s-1}}{x c n_{s-1}}\right)=\text { length }\left(x^{\prime} \mathfrak{a}^{*}\right)-\text { length } \mathfrak{a}^{*}=\text { length }\left(x^{\prime}\right)
$$

by Proposition 2. Combining our results we have

$$
\text { length }\left(\frac{\mathfrak{n}_{s-1}}{x \mathfrak{n}_{s-1}}\right)=\text { length }\left(x^{\prime}\right)
$$

and this establishes the theorem.

We can now combine Theorems 2 and 3 into the more comprehensive

ThEOREM 4. Let $Q$ be a one-dimensional local ring and let $x$ be an element of $m$ which is not a zero-divisor. Further, let

$$
Q 0=n_{1} \cap n_{2} \cap \ldots \cap n_{l}
$$

be a normal primary decomposition of the zero ideal, where $n_{i}$ is $p_{i}$-primary. If now $x_{i}$ denotes the residue of $x$ modulo $\mathfrak{p}_{i}$, then

$$
\text { length }(Q x)=\sum_{i=1}^{l}\left(\text { length } n_{i}\right) \text {. length }\left\{\left(Q / P_{i}\right) x_{i}\right\}
$$


4. Latent multiplicities and residue degrees. With the aid of Theorems 1 and 4 we shall now make a study of some of the properties of local domains.

Proposition 4. Let $Q$ be a complete one-dimensional local domain with quotient field $F$ and let $Q^{\prime}$ be the integral closure of $Q$ in $F$; then $Q^{\prime}$ is a finite $Q$-module. Further, $Q^{\prime}$ is a onedimensional regular local ring and therefore the valuation ring of a discrete rank 1 valuation.

Proof. The main difficulty, which consists in showing that $Q^{\prime}$ is a finite $Q$-module, $\dagger$ will be overcome by an appeal to the structure theory of local rings. $\quad$ First, if $Q$ has the same characteristic as its residue field, then $Q$ contains a subring $Q_{0}$ which is a ring of formal power series in one variable (with coefficients in a field) and over which $Q$ is a finite module. The only other alternative, since $Q$ is a domain, is that $Q$ should be of characteristic zero while its residue field is of characteristic $p \neq 0$. In this case, $Q$ contains a subring $Q_{0}$ having the following properties :

(i) $Q_{0}$ is a complete local domain whose maximal ideal is generated by $p$,

(ii) $Q$ and $Q_{0}$ are concordant and have the same residue field, which imply that $Q$ is a finite $Q_{0}$-module. Now, in either case, $Q_{0}$ is the valuation ring of a field $F_{0}$ with a complete discrete rank 1 valuation and the degree $\left[F: F_{0}\right]$ is finite; consequently, by a well-known result of Valuation Theory, the integral closure of $Q_{0}$ in $F$, namely $Q^{\prime}$, is a finite $Q_{0}$-module. It now follows, $a$ fortiori, that $Q^{\prime}$ is a finite $Q$-module and thus the main obstacle has been surmounted.

Since $Q^{\prime}$ is a finite $Q$-module, it follows, in the first instance, that $Q^{\prime}$ is a complete semilocal ring but, because $Q^{\prime}$ has no zero-divisors, we may go further and say that $Q^{\prime}$ is a local domain. Clearly $\operatorname{dim} Q^{\prime}=1$, and, since $Q^{\prime}$ is integrally closed, it follows that it must be regular. This completes the proof.

The valuation $v$ (say) determined by the valuation ring $Q^{\prime}$ will be said to be associated with $Q$, it being understood that $v$ is an additive valuation on $F$ and that its value group consists of all the positive and negative integers. If $\mathrm{m}^{\prime}$ is the maximal ideal of $Q^{\prime}$, then $Q^{\prime} / \mathrm{m}^{\prime}$ is the residue field of $Q^{\prime}$ and of $v$ and, moreover, the degree $\left[Q^{\prime} / \mathrm{m}^{\prime}: Q / \mathrm{m}\right]$ is finite. This degree will be referred to as the latent residue degree of $Q$. Both the notion of an associated valuation and of a latent residue degree will shortly be extended to local domains which are not complete. Before we proceed to do this, however, let us note that we have, as an immediate consequence of Theorem 1 ,

Proposition 5. Let $Q$ be a complete one-dimensional local domain with associated valuation $v$ and latent residue degree $f$. If now $x$ is a non-zero element of $Q$, then length $(Q x)=f v(x)$.

After these preliminaries, let $Q$ be a one-dimensional local domain which we no longer suppose to be complete. Denote by $\bar{Q}$ the completion of $Q$. Then not every non-unit of $\bar{Q}$ can be a zero-divisor ; consequently the zero ideal of $\bar{Q}$ has a normal decomposition

$$
\bar{Q} 0=n_{1} \cap n_{2} \cap \ldots \cap n_{l},
$$

where the $n_{i}$ are primary ideals belonging to different one-dimensional prime ideals $p_{i}$ (say). Let us now make the

DeFINITION. The numbers $\mu_{i}=$ length $n_{i}$, for $1 \leqslant i \leqslant l$, will be called the "latent multipli. cities" of the one-dimensional local domain $Q$.

$\dagger$ For a proof of the corresponding result for complete local domains of arbitrary dimension, see (6), Theorem 7, p. 118.

$\ddagger$ The structure theroems which are quoted here will all be found in the well known paper of I. S. Cohen. on the subject (3). For an interesting proof which avoids an appeal to the structure theory, see Krull (5). 
Note that if $Q$ is complete there is only one latent multiplicity and this has the value unity.

Consider now the ring $\bar{Q} / \mathcal{P}_{i}$. This is a complete one-dimensional local domain and, since $\dagger$ $P_{i} \cap Q=(0)$, it contains $Q$ as a subring. Indeed, $Q$ is not only a subring of $\bar{Q} / P_{i}$ but it is also everywhere dense in the ring.

Let $\bar{F}_{i}$ be the quotient field of $\bar{Q} / P_{i}$ and let $\bar{Q}_{i}^{\prime}$ be the integral closure of $\bar{Q} / P_{i}$ in $\overline{F_{i}}$; then, by Proposition $4, \bar{Q}_{i}^{\prime}$ is the valuation ring of a valuation $\bar{v}_{i}$. Morenver, $\bar{Q}_{i}^{\prime}$ and $\bar{Q} / P_{i}$ are, of course, concordant ; consequently $\bar{Q} / \rho_{i}$ is none other than the topological closure of $Q$ in $\bar{Q}_{i}^{\prime}$ or, equivalently, in the complete valuated field $\bar{F}_{i}$. This shows that if $v_{i}$ is the valuation induced by $\bar{v}_{i}$ on $F$, then $\bar{F}_{i}$ is the completion of $F$ with respect to $v_{i}$ and, moreover, we see that the value group of $v_{i}$ is the same as the value group of $\bar{v}_{i}$, namely the full additive group of integers. It is now convenient to make the

Defrnition. The valuations $v_{1}, v_{2}, \ldots, v_{l}$ induced on the quotient field $F$ of $Q$ will be called the "valuations associated with $Q$ ".

It should be observed that this agrees with the earlier terminology that was introduced in the case of a complete local domain, and we may also note that the valuations $v_{1}, v_{2}, \ldots, v_{2}$ are inequivalent. To see this, choose $\bar{z} \epsilon \bar{Q}$ so that $\dot{\bar{z}} \epsilon \mathcal{P}_{1}$ and $\bar{z} \notin \mathcal{P}_{2}$ and then choose a sequence $\left(q_{n}\right)$ of elements of $Q$ with the property that $q_{n} \rightarrow \bar{z}$ in $\bar{Q}$. This sequence will then tend to zero in $\bar{Q} / P_{1}$ and it will tend to a non-zero limit in $\bar{Q} / P_{2}$; consequently $v_{1}\left(q_{n}\right) \rightarrow \infty$ while $v_{2}\left(q_{n}\right)$ has a finite limit. This proves that $v_{1}$ and $v_{2}$ are inequivalent.

It will be convenient at this point to sum up some of our conclusions in

TheоRem 5. Let $Q$ be a one-dimensional local domain with quotient field $F$ and let $v_{1}, v_{2}, \ldots, v_{l}$ be the valuations of $F$ that are associated with $Q$. Then there is a $1-1$ correspondence between the valuations and the prime ideals $\mathcal{p}_{1}, \mathcal{P}_{2}, \ldots, \mathfrak{p}_{l}$ which belong to the zero ideal of the completion $\bar{Q}$ of $Q$. If $Q_{i}^{\prime}$ is the valuation ring of $v_{i}$ on $F$ and $\bar{Q}_{i}^{\prime}$ is its completion, then $Q \subseteq Q_{i}^{\prime}$ and the topological closure of $Q$ in $\bar{Q}_{i}^{\prime}$ can be identified with $\bar{Q} / P_{i}$. On this understanding $\bar{Q}_{i}^{\prime}$ is a finite $\bar{Q} / P_{i}$-module and the two rings have the same quotient field.

Still keeping to the same notations, let $x$ be a non-zero element of the maximal ideal of $Q$; then

$$
\text { length } \left.(Q x)=\text { length }(\bar{Q} x)=\Sigma \text { (length } n_{i}\right) \text { length }\left\{\left(\bar{Q} / \mathcal{P}_{i}\right) x\right\},
$$

by Theorem 4 ; consequently, using Proposition 5 ,

$$
\text { length }(Q x)=\sum_{i=1}^{l} \mu_{i} f_{i} \bar{v}_{i}(x)=\sum_{i=1}^{l} \mu_{i} f_{i} v_{i}(x) \text {, }
$$

where $f_{i}$ is the latent residue degree associated with $\bar{Q} / \mathfrak{p}_{i}$. Now $f_{i}$ is simply the degree of the residue field of $\bar{v}_{i}$ over the residue field of $\bar{Q} / P_{i}$ and this, in turn, is none other than the degree of the residue field of $v_{i}$ over $Q / \mathrm{m}$. Accordingly, we make the

DEFINITION. If $v_{1}, v_{2}, \ldots, v_{l}$ are the valuations associated with the one-dimensional local domain $Q$, then the residue field of $v_{i}$ has a finite degree $f_{i}$ over the residue field of $Q$. The numbers $f_{i}$ will be called the "latent residue degrees" of $Q$.

With the aid of this definition we can now restate (4.1) as

Theorem 6. Let $Q$ be a one-dimensional local domain, let $\mu_{i}$ be the latent multiplicities and $f_{i}$ the latent residue degrees, where $1 \leqslant i \leqslant l$. Further, let $v_{i}(1 \leqslant i \leqslant l)$ be the associated valuations.

$\dagger$ Because no non-zero element of $Q$ is a zero-divisor in $\bar{Q}$. 
If now $x$ is a non-zero element of $Q$, then

$$
\text { length }(Q x)=\sum_{i=1}^{l} \mu_{i} f_{i} v_{i}(x)
$$

Remark. It is worth while noting, for convenience in applications, that we do not have to exclude the possibility that $x$ may be a unit of $Q$.

5. A direct construction of the associated valuations. It will be noticed that our construction of the associated valuations, which begins with the simplified construction available in the case of the complete rings $\bar{Q} / \mathcal{P}_{i}$, is very indirect. It has, however, the advantage of by-passing the main difficulty that presents itself if one attempts to proceed in a less circuitous manner, $\dagger$ and we can now fill in the gaps in the theory without much trouble. This will be the object of the present section, and the terminology used will be that introduced in the part of $\$ 4$ that follows Proposition 5 .

LEMMA 1. Let $Q$ be a one-dimensional local domain and $v_{1}, v_{2}, \ldots, v_{l}$ the associated valuations on its quotient field $F$. Further, let $A$ be the integral closure of $Q$ in $F$. If now $y$ is an element of $F$, then $y$ belongs to $\Lambda$ if, and only if, $v_{i}(y) \geqslant 0$ for $1 \leqslant i \leqslant l$.

Proof. By Theorem $5, Q$ is contained in the valuation ring of $v_{i}$; consequently, since valuation rings are integrally closed, $A$ is also contained in the valuation ring of $v_{i}$. This proves half the lemma. Now suppose that $y=a / b$, where $a$ and $b$ are in $Q$ and that $v_{i}(y) \geqslant 0$ for $1 \leqslant i \leqslant l$. It remains for us to show that $y$ is integral with respect to $Q$.

Since $v_{i}(y) \geqslant 0, y$ belongs to the valuation ring $\bar{Q}_{i}^{\prime}$ of the completion of $F$ with respect to $v_{i}$; consequently, since $\bar{Q}_{i}^{\prime}$ is a finite $Q / \rho_{i}$-module, $y$ is integral with respect to $\bar{Q} / \rho_{i}$. It follows, therefore, that we have a relation of the form

$$
a^{n_{i}}+\tau_{1}^{(i)} a^{n_{i}-1} b+\tau_{2}^{(i)} a^{n_{i}-2} b^{2}+\ldots+\tau_{n_{i}}^{(i)} b^{n_{i}}=0,
$$

where $\tau_{j}^{(i)} \in \bar{Q} / \rho_{i}$. If now $\bar{t}_{j}^{(i)}$ is a representative of $\tau_{j}^{(i)}$ in $\bar{Q}$, then

$$
a^{n_{i}}+\bar{t}_{1}^{(i)} a^{n_{i}^{-1}} b+\bar{t}_{2}^{(i)} a^{n_{i}-2} b^{2}+\ldots+\bar{t}_{n_{i}}^{(i)} b^{n_{i}} \equiv 0\left(\bmod p_{i}\right)
$$

We have, of course, a congruence of this kind for each value of $i$ and, moreover, $\mathcal{P}_{1} \cap \mathcal{P}_{2} \frown \ldots \frown \mathcal{P}_{1}$ consists entirely of nilpotent elements. If, therefore, we multiply together the left hand sides of these congruences and raise the result to a high enough power we arrive at a relation

where $\bar{t}_{i} \in \bar{Q}$. Thus

$$
a^{n}+\bar{t}_{1} a^{n-1} b+\bar{t}_{2} a^{n-2} b^{2}+\ldots+\bar{t}_{n} b^{n}=0,
$$

which shows that

$$
\begin{aligned}
a^{n} & \in Q \cap\left(\bar{Q} a^{n-1} b+\bar{Q} a^{n-2} b^{2}+\ldots+\bar{Q} b^{n}\right) \\
& =Q \cap \bar{Q}\left(Q a^{n-1} b+Q a^{n-2} b^{2}+\ldots+Q b^{n}\right) \\
& =Q a^{n-1} b+Q a^{n-2} b^{2}+\ldots+Q b^{n}
\end{aligned}
$$

$$
a^{n}+q_{1} a^{n-1} b+q_{2} a^{n-2} b^{2}+\ldots+q_{n} b^{n}=0,
$$

where $q_{i} \in Q$. If now we divide through the equation by $b^{n}$, the integral dependence of $y=a / b$ on $Q$ becomes apparent.

LEMMA 1 shows that $\Lambda$ is the intersection of the valuation rings of $v_{1}, v_{2}, \ldots, v_{l}$ and, as

+ The difficulty arises from the fact that the integral closure of $Q$ in its quotient field may not be a tinite $Q$-module. 
is well-known, this implies that $\Lambda$ is a principal ideal domain having only a finite number of proper prime ideals. These facts and a few others, which are immediate consequences of them, are set out in

Theorem 7. $\dagger$ Let $Q$ be a one-dimensional local domain and let $\Lambda$ be its integral closure in its quotient field $F$. Then $\Lambda$ is a principal ideal domain and it has only a finite number $\mathfrak{p}_{1}, \mathfrak{p}_{2}, \ldots, \mathfrak{p}_{2}$ of non-trivial prime ideals. The rings of quotients $\Lambda_{\mathfrak{p}_{i}}$ are the valuation rings of the valuations that are associated with $Q$. Finally, the degrees $\left[\Lambda / \mathfrak{p}_{i}: Q / \mathrm{m}\right]$ are all finite and these degrees are none other than the latent residue degrees that are associated with $Q$.

6. Analytically unramified rings. The generality of Theorems 6 and 7 is, to some extent, indicated by the fact that we have not had to assume that the integral closure $A$ of $Q$ in $F$ is a finite $Q$-module. It is true that this condition is satisfied in certain important special cases $\ddagger$ but, from the point of view of a general theory, to impose it would be to make an undesirable restriction. We can see just what this restriction would entail from

Theorem 8. Let $Q$ be a one-dimensional local domain and let $\Lambda$ be the integral closure of $Q$ in its quotient field $F$. Then the following assertions are equivalent :

(i) $A$ is a finite $Q$-module,

(ii) $Q$ is a subspace of $A$ if the latter is regarded as a semi-local ring,

(iii) The zero ideal of the completion $\bar{Q}$ of $Q$ is an intersection of prime ideals,

(iv) The latent multiplicities of $Q$ are all unity.

We recall that a local domain having the property (iii) is said to be analytically unramified.

Proof. Assume that (i) is satisfied. Then the conductor f from $Q$ to $\Lambda$ is non-zero and therefore either $f=Q$-in which case $A=Q$ and (ii) is obvious-or else, as we shall suppose, $f$ is m-primary. Let $p_{1}, p_{2}, \ldots, p_{l}$ be the maximal ideals of $\Lambda$; then $m \subseteq p_{1} p_{2} \ldots p_{l}$ and the $\mathfrak{p}_{i}$ all belong to $f$ when it is regarded as a $A$-ideal. We can therefore find $t$ so that

$$
\left(p_{1} p_{2} \ldots p_{l}\right)^{t} \subseteq f \subseteq m \subseteq p_{1} p_{2} \ldots p_{l}
$$

and this shows that $Q$ is a subspace of $\Lambda$. Thus (i) implies (ii).

Suppose now that $Q$ is a subspace of $\Lambda$; then $\bar{Q}$ is contained in the completion $\bar{\Lambda}$ of $\Lambda$. But $\bar{\Lambda}$ is a direct sum of integral domains ; consequently the only nilpotent element in $\bar{\Lambda}$, and therefore also in $\bar{Q}$, is the zero element. This means, however, that the zero ideal of $\bar{Q}$ is an intersection of prime ideals. Thus (ii) implies (iii) and (iii), of course, is equivalent to (iv).

To complete the proof, we assume that

$$
\bar{Q} 0=\mathcal{P}_{1} \frown \mathcal{P}_{2} \frown \ldots \frown \mathcal{P}_{l},
$$

where the $p_{i}$ are one-dimensional prime ideals, and deduce that $\Lambda$ is a finite $Q$-module. $\|$ Let $S$ be the full ring of quotients of $\bar{Q}$; then, as in $\S 2$, we can find elements $e_{1}, e_{2}, \ldots, e_{1}$ such that $e_{i} \equiv 1\left(\bmod S \mathcal{P}_{i}\right)$ and $e_{i} \equiv 0\left(\bmod S \mathcal{P}_{j}\right)$ if $i \neq j$. These elements are orthogonal idem. potents, $e_{1}+e_{2}+\ldots+e_{l}=1$ and

$$
S p_{i}=S e_{1}+\ldots+S e_{i-1}+S e_{i+1}+\ldots+S e_{l} .
$$

Accordingly $S / S P_{i}$ can be identified with $S e_{i}$ and $\bar{Q} / \mathcal{P}_{i}$ with $\bar{Q} e_{i}$. But $S / S \mathcal{P}_{i}$ is the quotient

† For a simple direct proof that every ring between $Q$ and $\Lambda$ is Noetherian, see Chevalley (2), p. 32. This result is due originally to Akizuki (1).

$\mp$ For example, it is always satisfied if $Q$ is the local ring of a point on a curve.

1) For a generalization of this to local domains of any dimensions, see Nagata (7), Lemma 13, p. 16. 
field of $\bar{Q} / \rho_{i}$; consequently, by Proposition 4 , the integral closure of $\bar{Q} e_{i}$ in $S e_{i}$ is a finite $\bar{Q} e_{i}$-module, say

$$
\left(\bar{Q} e_{i}\right) \omega_{1}^{(i)}+\left(\bar{Q} e_{i}\right) \omega_{2}^{(i)}+\ldots+\left(\bar{Q} e_{i}\right) \omega_{n}^{(i)} .
$$

Here, for convenience of notation, we have arranged (as we clearly may) that the number $n$ of elements in the module base is independent of $i$.

Now let $P$ be the integral closure of $\bar{Q}$ in $S$ and let $\pi \in P$; then $\pi e_{i}$ is integral with respect to $\bar{Q} e_{i}$ and so belongs to the module (6.1). But $\pi=\pi e_{1}+\pi e_{2}+\ldots+\pi e_{l}$; consequently $\pi$ belongs to

$$
\sum_{i, j} \bar{Q} e_{i} \omega_{j}^{(i)}
$$

and therefore $P$ is contained in (6.2). Choose $\bar{c} \epsilon \bar{Q}$ so that $\bar{c}$ is not a zero-divisor and $P \bar{c} \subseteq \bar{Q}$; then, for a suitable integer $h, \overline{\mathrm{m}}^{h} \subseteq \bar{Q}_{\bar{c}}$ and therefore

$$
P \bar{\pi}^{n} \subseteq P \bar{c} \subseteq \bar{Q} \text {. }
$$

Here, of course, $\bar{m}$ is the maximal ideal of $\bar{Q}$. Next choose $c \in Q$ so that $c \neq 0$ and $c \equiv \bar{c}$ (mod $\left.\overline{\mathrm{m}}^{n}\right)$; then $P c \subseteq P \bar{c}+P \overline{\mathrm{m}}^{n} \subseteq \bar{Q}$. Finally, let $\lambda=a / b$ be an element of $\Lambda$, where $a$ and $b$ are in $Q$; then, as an element of $\subseteq, \lambda$ is integral with respect to $\bar{Q}$ and so $\lambda \in P$. From $P_{c} \subseteq \bar{Q}$ we now obtrin $a c \epsilon \bar{Q} b \cap Q=Q b$ and so $\lambda \epsilon Q c^{-1}$. Thus $\Lambda$ is a submodule of a finite $Q \cdot$ module and thereforc, because $Q$ is Noetherian, $\Lambda$ is itself a finite $Q$-module.

\section{REFERENCES}

(1) Y. Akizuki, Einige Bemerkungen über primäre Integritätsbereiche mit Teilerkettensatz, Proc. Phys.-Math. Soc. Japan. 17 (1935), 327-336.

(2) C. Chevalley, La notion d'anncau de décomposition, Nagoya Math. J. 7 (1954), 21-33.

(3) I. S. Cohen, On the structure and ideal theory of complete local rings, Trans. Amer. Math. Soc. 59 (1946), 54-106.

(4) H. Grell, Verzweigungstheorie in allgemeinen Ordnungen algebraischer Zahlkörper, Math. Z., $40(1935), 629-657$.

(5) W. Krull, Ein Satz über primäre Integritätsbereiche, Math. Ann. 103 (1930), 450-465.

(6) M. Nagata, Some remarks on Local Rings II, Memoirs of College of Science, Kyoto Univ. 28, Mathematics No. 2 (1953), 109-120.

(7) M. Nagata, On the theory of Henselian rings II, Nagoya Math. J. 7 (1954), 1-19.

(8) D. G. Northcott, Ideal Theory, Cambridge Tracts No. 42.

(9) P. Samuel, La notion de multiplicité en algèbre et en géométrie, Thesis, Paris (1951).

(10) R. J. Walker, Algebraic Curves (Princeton Math. Series No. 13).

The UnIversity,

SheFFIELD. 